\title{
Determination Of Curcumin In Capsule Containing Curcuma xanthorrhiza Roxb. Extracts By High Performance Liquid Chromatography
}

\author{
Firdaus Candra Kurniawan \\ Analytical Development Scientist, Research And Development Department \\ Pt. Dexa Medica, Cikarang, Bekasi, Jawa Barat \\ Email : Fckurniawan_Apt@Yahoo.Com
}

\begin{abstract}
Curcumin, the major yellow pigment of Curcuma sp. has several interesting biological activities, such as anticarcinogenecity, antiinflamatory, and antioxidant. Curcumin naturally occurs as major compound in Curcuminoid substance beside its derivatives, i.e. Bisdemethoxycurcumin (BDC) and Demethoxycurcumin (DC). Quantitative analysis of Curcumin is difficult to carry out unless prior to completely resolved Curcumin from BDC and DC. A good resolution, reproducible, and accurate method for determination Curcumin in pharmaceutical product (capsule formed) by HPLC has been carry out. Using $\mathrm{C}_{18}$ colurnn and mixture of Acetonitrile: Acetic Acid glacial 1\% (45:55 v/v) as eluent, BDC, DC, and Curcumin were retained with retention time of $10.79,12.29$, and 13.91 minutes, respectively. Curcumin was simultaneously separated from BDC or DC. The resolution factor (Rs) between Curcumin and DC was 1.73. Good linear relationship $(\mathrm{r}=0.9997, \mathrm{Vxo}=0.99 \%)$ between peak area and concentrations was found at range of the Curcumin concentration used, i.e. 23.56-62.83 $\mu \mathrm{g} /$ $\mathrm{mL}$. Curcumin recoveries were (93.60-118.17)\% with the coefficient of variation (CV) 6.99\%. Curcumin content in each capsule sample was found to be $(8.89 \pm 0.06) \mathrm{mg}$. It showed that samples contained higher amount of Curcumin than that claimed on its label, $5 \mathrm{mg}$.
\end{abstract}

Keywords: Bisdemethoxycurcumin, Demethoxycurcumin, Curcumin, Curcuminoid, HPLC.

\section{INTRODUCTION}

Curcumin [7-Bis-(4-hydroxy-3methoxyphenyl)-hepta-1,6-dien-3,5-dion] (Fig. 1) is a useful substance for human health. Curcumin naturally occurs as major compound in Curcuminoid substance besides its derivatives, i.e. Bisdemethoxycurcumin (BDC) and Demethoxycurcumin (DC). Curcumin have several interesting biological activities, such as anticarcinogenecity, antiinflamatory, and antioxidant (Verghese, 1999; Majeed, et al., 2000; Stankovic, 2004). Curcumin content in registered pharmaceutical product must be controlled in order to guarantee whether its concentration conforms to label's declared. Curcumin as a natural product is difficult to be measured quantitatively unless completely resolved from BDC and DC.

The aim of this study was to develop a HPLC method for determination of Curcumin in a registered pharmaceutical product (capsule formed).

\section{MATERIALS AND METHOD}

Materials

Curcumin (Sigma-Aldrich, which has $77.1 \%$ purity by HPLC) was used as 
received from its manufacturer for preparing standard solution in methanol. Curcumin's Certificate of Analysis declared that Standard Curcumin was extracted from Curcuma

longa (Turmeric). Standard Curcumin solutions were prepared daily $(r p)$.

\section{Chemicals}

Methanol and Acetonitrile (J.T. Baker) were HPLC grade. Acetic acid (Merck) was pro analysis grade.

\section{Instrument}

HPLC Agilent 1100 series equipped with Lichrospher RP 18 column and Diode Array Detector.

\section{Sample}

Temulawak capsule which has registration number DEPKES RI No. TR: 963385001. The declared content of each capsule was Curcumae xanthorrhizae Roxb. Extract equivalent to $5 \mathrm{mg}$ Curcumin.

\section{Sample Preparation}

Ten capsules were opened in order to determine the average weight of their powder content. The powder obtained was homogenized. Powder (ca. $265 \mathrm{mg}$ ) was weighted accurately and transferred into a $50 \mathrm{~mL}$ volumetric flask. Approximately 20 $\mathrm{mL}$ of methanol was added and the mixture was placed in vortex agitator for 3 minutes. Furthermore, the flask was filled with methanol up to $50.0 \mathrm{~mL}$ and shaken homogenously. A mixture obtained was filtered through a Whatman No. 40 filter paper. The first milliliter filtrate was discarded. A suitable amount of filtrate was diluted quantitatively twice or four times with methanol before it was filtered through $0,2 \mu \mathrm{m}$ Milipore filters. Twenty microliter of diluted filtrate was ready to be injected into HPLC system.

\section{Validation of the Method}

Optimum condition of HPLC system for separation Curcumin from BDC and DC was obtained by modiffing various eluent mixture and eluent flow rate. Identification of Curcumin peak in sample was based on similarity of retention time $\left(t_{R}\right)$, peak's profile, and overlapping of peak's spectra (using match factor value) compared to standard Curcumin. Selectivity parameter was Resolution factor (Rs) between Curcumin and other substances in sample.

The accuracy of the method was validated by spiking samples with standard Curcumin at three fortification level (50$150) \%$ of expected value. Each concentration level was analyzed triplicate (Cunnif, 1995; USP XXVI, 2003). The precision was evaluated by analyzing nine values of percentage recovery obtained in determination of accuracy.

The linearity over the range investigated can be assessed from graph of Standard

Curcumin concentration versus area obtained (five times replications).

\section{RESULT AND DISCUSSION}

\section{Separation of Curcumin from Its Derivatives}

Figure 2 shows the absorption spectra of 1 and $2.5 \mu \mathrm{g} / \mathrm{mL}$ standard Curcumin solution. The $\ddot{\mathrm{e}}_{\max }$ of Curcumin(420 nm) was then selected for quantitative analysis.

Optimum HPLC system for the separation of Curcumin from other substances was obtained by eluted sample through a mixture of Acetonitrile: Acetic 
<smiles>[R]c1cc(/C=C/C(=O)CC(=O)/C=C/c2ccc(O)c([R])c2)ccc1O</smiles>

Figure 1. Structure of Curcuminoid

Curcumin

Demethoxycurcumin (DC)

Bisdemethoxycurcumin (BDC) $\mathrm{R}_{1}$ and $\mathrm{R}_{2}=-\mathrm{H}$

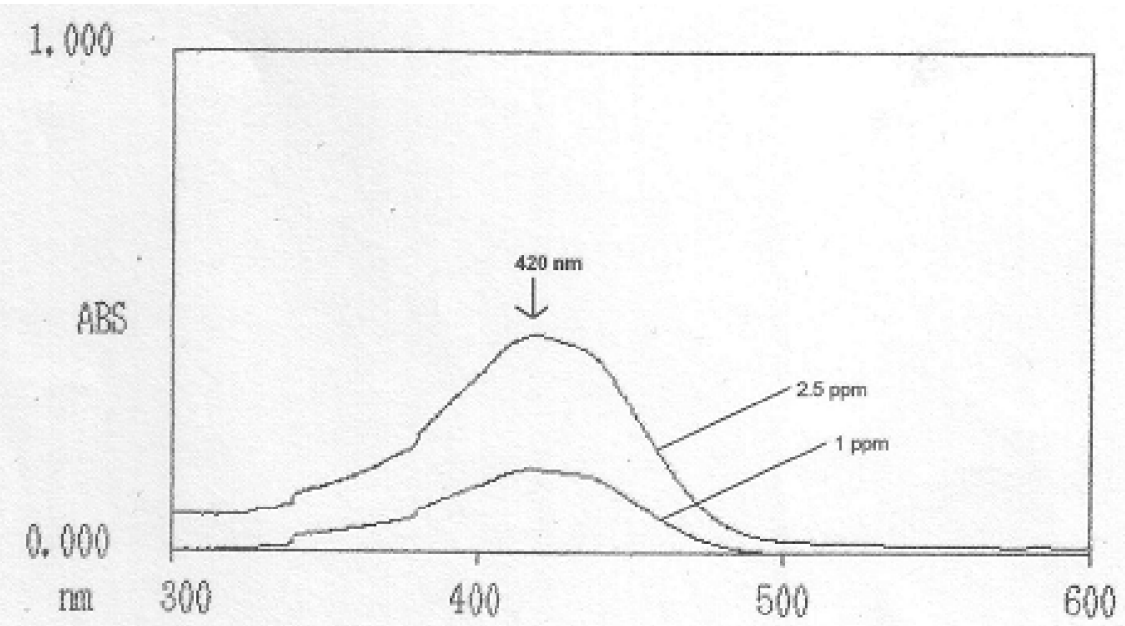

Figure 2. Spectra of Standard Curcumin Solution in Methanol (Using Perkin Elmer Spectrophotometer)

Acid glacial 1\% (ratio: 45:55 v/v). The optimum eluent flow rate was $1.0 \mathrm{ml} /$ minute.

Figure 3 shows three peaks obtained from chromatogram of standard Curcumin with retention time $\left(t_{R}\right)$ of $10.7,12.2$, and 13.9 minutes, respectively. According to Naoki et al (2003), standard Curcumin which is obtained by extraction from Curcuma longa generally contains BDC and DC. Percentage area of the third peak was $77.01 \%$. It was in conformity with Curcumin's Certificate of Analysis that the third peak was Curcumin. The first and second peak were supposed to be BDC and
DC, respectively. Curcumin can be resolved from DC with Rs value of 1.73.

Figure 4 shows HPLC chromatogram of Temulawak capsule sample. That chromatogram shows two significant peaks, which have $t_{R}$ of 12.3 rninutes and 13.9 minutes respectively. The second peak was supposed to be curcumin based on its similmity of retention time $\left(t_{R}\right)$, peak's profile, and peak's spectra profile (has matching factor value of 999.93) compared to standard Curcumin.

Chromatogram of sample extract that was spiked with standard Curcumin can be seen on Fig. 5. The supposed Curcumin's 


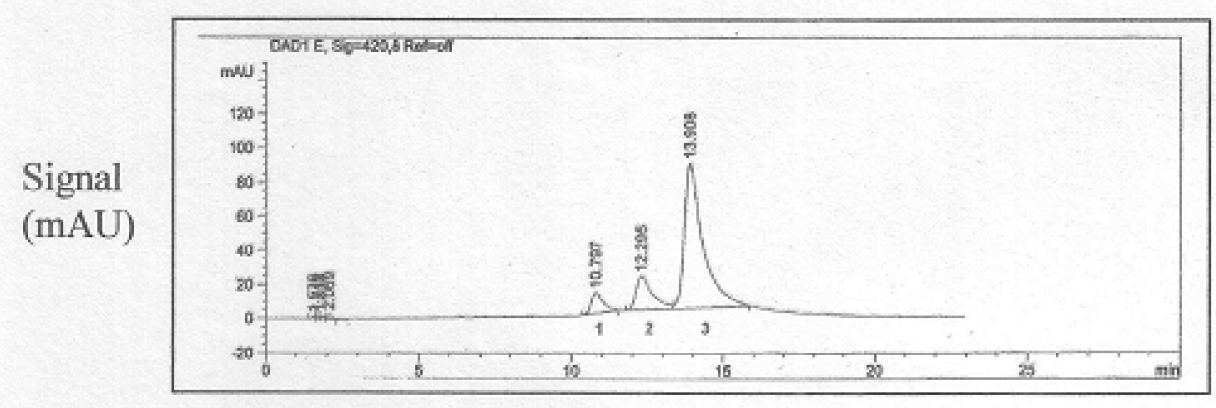

Retention Time (min)

Figure 3. Chromatogram of Standard Curcumin

$1=\mathrm{BDC}, 2=\mathrm{DC}, 3=$ Curcumin

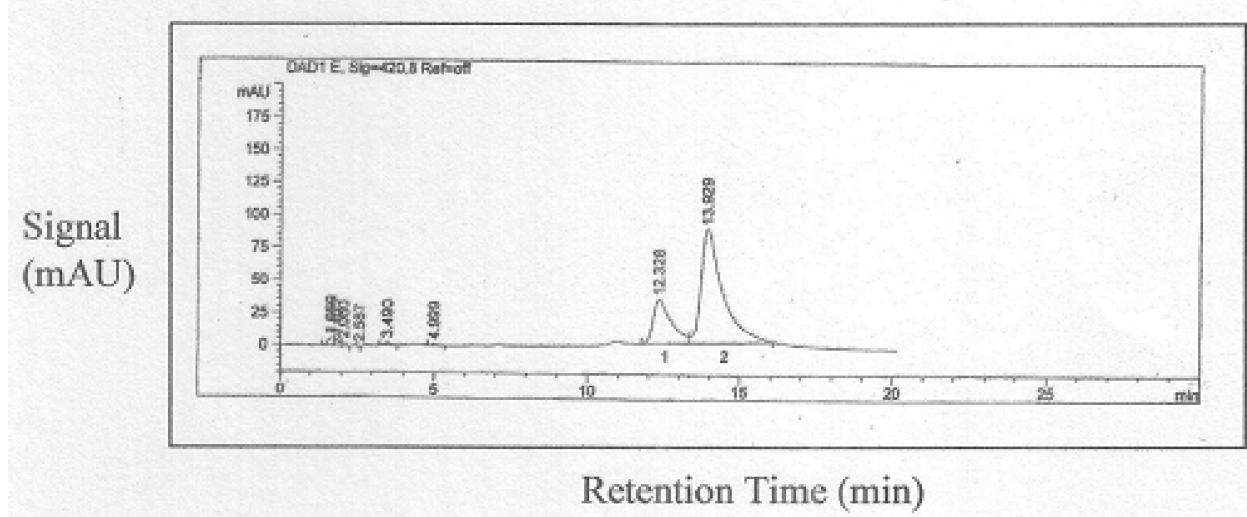

Figure 4. Chromatogram of Temulawak Capsule Sample $1=\mathrm{DC}, 2$ = Curcumin

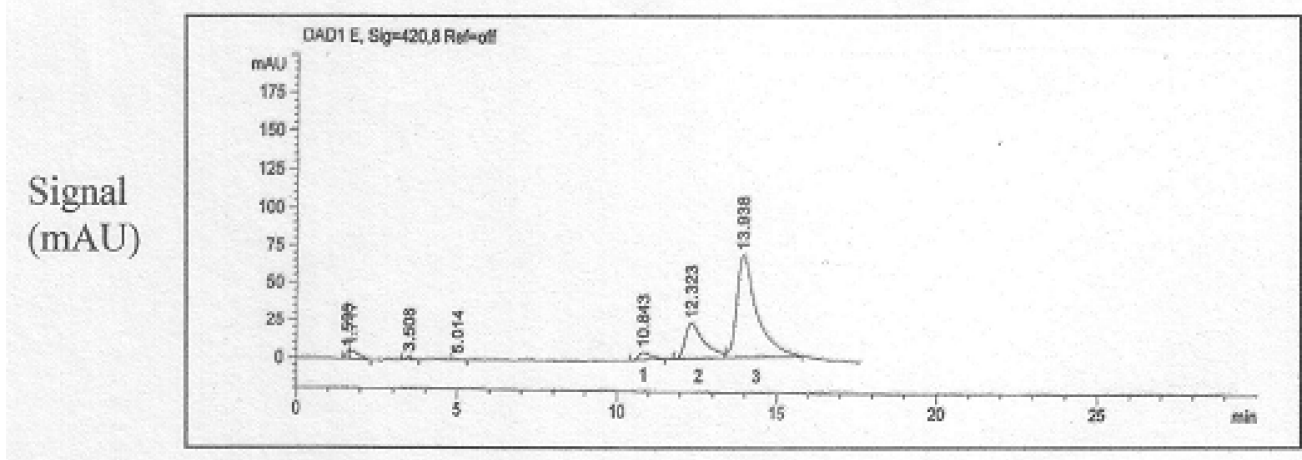

Retention Time (min)

Figure 5. Chromatogram of Sample's Extract Spiked with Standard Curcumin $1=\mathrm{BDC}, 2=\mathrm{DC}, 3=$ Curcumin 
peak consistently showed its similarity of retention time $\left(t_{R}\right)$, peak's profile, and peak's spectra profile compared to standard Curcumin.

\section{Linearity}

According to linearity test that had been established, Curcumin concentrations in the range of $(23.56-62.83) \mu \mathrm{g} / \mathrm{mL}$, showed a linearity correlation with its relative response of detector (peak area). Coefficient correlation ( $\mathrm{r}_{\text {calculated }}$ ) was 0.9997 , more than $\mathrm{r}_{\text {table }}$ of 0.9000 (for $\mathrm{p}=$ 0.95 and $n=5$ ). Linear regression equation was $\mathrm{y}=126.4 \mathrm{X}-1287.8$ with relative standard deviation of function $\left(\mathrm{V}_{\mathrm{xo}}\right) 0.99 \%$.

\section{Precision and Accuracy}

Accuracy of the method was validated by spiking sample's extract with standard Curcumin at three concentration level 23.56, 39.27, and $47.12 \mu \mathrm{g} / \mathrm{mL}$. Each concentration was analyzed triplicate. Recoveries of standard Curcumin concentration added were at the range of (93.60-118.17)\% with CV 6.99\%. The recoveries of standard Curcumin added were shown on table 1 .

Instrument precision was determined by injecting 10 times of each 20,0 $\mu \mathrm{L} 50 \mu \mathrm{g} / \mathrm{mL}$ standard Curcumin solution into HPLC system. The coefficient of variation $(\mathrm{CV})$ of peak area observed $(1.19 \%)$ showed the reproducibility of instrument in determining peak area of Curcumin was good. Precision of the method was studied by assaying nine samples spiked with standard Curcumin of 23.56, 39.27, and $47.12 \mu \mathrm{g} / \mathrm{mL}$. The coefficient of variation $(\mathrm{CV})$ of percentage of standard Curcumin recoveries $6.99 \%$ was good.

\section{Analysis of the Sample}

The concentrations of Curcumin in Temulawak capsule sample obtained were in the range of (8.836-8.995) $\mathrm{mg}$ as reported in table2.The Curcumin concentration in sample was higher (177\%) than that mentioned

in its label (5 mg per capsule).

\section{CONCLUSIONS}

The method that was used for determining Curcumin content in pharmaceutical product (capsule formed) fulfilled with the standard validation criteria. The Curcumin content in each capsule sample was found to be $(8.89 \pm 0.06) \mathrm{mg}$. It showed that samples contained higher amount of Curcumin than that claimed on its label, $5 \mathrm{mg}$.

\section{REFERENCES}

1. Cunil, P., 1995. Official Methods of Analysis of International AOAC, $16^{\text {th }}$ Ed. USA: AOAC International Suite 400.

2. Majeed, M., Badmaev, V., Shivakumar,

U.\& Rajendran, R., 2000.

Curcuminoids: Antioxidant

Phytonutrients, Sabinsa

Corporation.

3. Naoki A., Masanori T., Teiichi H., Masaaki, U., Aishin S., Yasuo M., Kengo K., 2003. Determination of Curcumin Content of Turmeric by High Performance Liquid Chromatography. Journal of The Pharmaceutical Society of Japan, Vol.101, p.374-77.

4. Sasaki, S.S., Sat, K., Abe, M., Sugimoto, N., and Maitani, T., 1998. 
Components of Turmeric Oleoresin Preparations and Photo-stability of Curcumin Japan. Journal Food Chem, Ed.5, Vol.1. Available at: http://www.jpfsc.com (accesed at 17 January 2008).

5. Stankovic, Ivan, 2004. Curcumin, Chemical and Technical Assessment (CTA). 61 $1^{\text {st }} \mathrm{JECF}$. Available at: ftp://ftp.fao.org/es/esn/ jecfalcta/CTA_61_Curcumin.pdf (accesed at 17 January 2008).

6. The United States Pharmacopoeia National Formulary, 2003. Ed.XXVI, United States Pharmacopeial Convention, Inc., Rockville, MD.

7. Tonnesen, H.H. and Karlsen, J., 1983.

High Perforrnance Liquid Chromatography of Curcumin and Related Components. J. Chromatography, Vol.259, p.36771.

8.Verghese, J., 1999. Curcuminoids, the Magic Dye of C. longa L. rhizome. Indian Spices, Vol.36(4), p.19-26. 